\title{
On repdigits as product of consecutive Lucas numbers
}

\author{
Nurettin Irmak ${ }^{1}$ and Alain Togbé ${ }^{2}$ \\ ${ }^{1}$ Mathematics Department, Art and Science Faculty \\ Niğde Ömer Halisdemir University, Niğde, Turkey \\ e-mails: irmaknurettin@gmail.com, nirmak@ohu.edu.tr \\ 2 Department of Mathematics, Purdue University Northwest \\ 1401 S. U. S. 421., Westville, IN 46391, United States \\ e-mail: atogbe@pnw. edu
}

Abstract: Let $\left(L_{n}\right)_{n \geq 0}$ be the Lucas sequence. D. Marques and A. Togbé [7] showed that if $F_{n} \ldots F_{n+k-1}$ is a repdigit with at least two digits, then $(k, n)=(1,10)$, where $\left(F_{n}\right)_{\geq 0}$ is the Fibonacci sequence. In this paper, we solve the equation

$$
L_{n} \ldots L_{n+k-1}=a\left(\frac{10^{m}-1}{9}\right) \text {, }
$$

where $1 \leq a \leq 9, n, k \geq 2$ and $m$ are positive integers.

Keywords: Lucas numbers, Repdigits.

2010 Mathematics Subject Classification: 11A63, 11B39, 11B50.

\section{Introduction}

Let $\left(F_{n}\right)_{n \geq 0}$ be the Fibonacci sequence given by the relation $F_{n}=F_{n-1}+F_{n-2}$, for $n \geq 2$ and with $F_{0}=0, F_{1}=1$. Its companion sequence is known as Lucas sequence $\left(L_{n}\right)_{n \geq 0}$ that satisfies the same relation with Fibonacci sequence together with the initial conditions $L_{0}=2$ and $L_{1}=1$. These numbers have very amazing properties (for see details, we refer the book of Koshy [3]).

Finding special properties in these sequences is a very interesting problem. The most famous one is given by Bugeaud et al. [1] as that the only perfect powers in Fibonacci sequence are 
0, 1, 8 and 144. Luca and Shorey [6] proved that product of consecutive Fibonacci numbers is not a perfect power of exponent larger than one of an integer except the trivial case $F_{1} F_{2}=1$.

If a positive integer has only one distinct digit in its decimal expansion, then we call it "repdigit". Obviously, such a number has the form $a\left(10^{m}-1\right) / 9$, for some $m \geq 1$ and $1 \leq a \leq 9$. It is natural to ask, which numbers are repdigits in Fibonacci and Lucas sequences? This question was answered by Luca [5] in 2000 by showing that 55 is the largest repdigit Fibonacci number and 11 is the largest repdigit Lucas number. Recently, Marques and Togbé [7] proved the following result.

Theorem 1. The only solution of the Diophantine equation

$$
F_{n} \cdots F_{n+(k-1)}=a\left(\frac{10^{m}-1}{9}\right)
$$

in positive integers $n, k, m, a$, with $1 \leq a \leq 9$ and $m>1$ is $(n, k, m, a)=(10,1,2,5)$.

For the proof of the above theorem, they used mathematical induction, Fibonacci recurrence pattern, congruence properties, etc. But, the main point of their proof is the identity $5 \mid F_{5 n}(n \geq 0)$.

The aim of this paper is to study a similar problem but in the case of Lucas numbers. Our main result is as follows.

Theorem 2. The quadruple $(n, k, m, a)=(4,2,2,7)$ is the only solution of the Diophantine equation

$$
L_{n} \ldots L_{n+k-1}=a\left(\frac{10^{m}-1}{9}\right)
$$

for some $m \geq 1, k \geq 2$ and $1 \leq a \leq 9$ being integers.

In order to solve the equation, we use the definition of $p$-adic order of an integer, linear forms in logarithms à la Baker, and congruence properties.

\section{Auxiliary results}

The $p$-adic order of $r$ is the exponent of the highest power of a prime $p$ which divides $r$. We denote it by $\nu_{p}(r)$. Now, we recall a result of Lengyel [4] on the 2-adic order of a Lucas number.

Lemma 1. For $n \geq 0$ integer, then

$$
\nu_{2}\left(L_{n}\right)=\left\{\begin{array}{ccc}
0, & n \equiv 1,2 & (\bmod 3) \\
2, & n \equiv 3 \quad(\bmod 6) \\
1, & n \equiv 0 \quad(\bmod 6) .
\end{array}\right.
$$

The Binet formula for a Lucas number is

$$
L_{n}=\alpha^{n}+\beta^{n}
$$

where $\alpha(>1)$ and $\beta(<1)$ are the roots of the characteristic equation $x^{2}-x-1=0$. Moreover, we have

$$
\alpha^{n-1}<L_{n}<\alpha^{n+1}, \quad \text { for } n \geq 0 .
$$


Since we use Baker method for our proof, we give two lemmata due to Matveev [8] (and Theorem 9.4 of [1]), Dujella and Pethö [2]. It is well-known that the logarithmic height of an algebraic number $\eta$ is defined as

$$
h(\eta)=\frac{1}{d}\left(\log a_{0}+\sum_{i=1}^{d} \log \left(\max \left\{\left|\eta^{(i)}, 1\right|\right\}\right)\right),
$$

where $d$ is the degree of $\eta$ over $\mathbb{Q}$ and $\left(\eta^{(i)}\right)_{1 \leq i \leq d}$ are the conjugates of $\eta$ over $\mathbb{Q}$, and $a_{0}$ is the leading coefficient of the irreducible polynomial of $\eta$.

Lemma 2. Let $\mathbb{K}$ be a number field of degree $D$ over $\mathbb{Q}, \gamma_{1}, \gamma_{2}, \ldots, \gamma_{t}$ be positive real numbers of $\mathbb{K}$, and $b_{1}, b_{2}, \ldots, b_{t}$ nonzero rational integers. Put

$$
B \geq \max \left\{\left|b_{1}\right|,\left|b_{2}\right|, \ldots,\left|b_{t}\right|\right\}
$$

and

$$
\Lambda:=\gamma_{1}^{b_{1}} \cdots \gamma_{t}^{b_{t}}-1
$$

Let $A_{1}, \ldots, A_{t}$ be positive real numbers such that

$$
A_{i} \geq \max \left\{D h\left(\gamma_{i}\right),\left|\log \gamma_{i}\right|, 0.16\right\}, \quad i=1, \ldots, t .
$$

Then, assuming that $\Lambda \neq 0$, we have

$$
|\Lambda|>\exp \left(-1.4 \times 30^{t+3} \times t^{4.5} \times D^{2} \times(1+\log D)(1+\log B) A_{1} \ldots A_{t}\right) .
$$

Lemma 3. Suppose that $M$ is a positive integer. Let $p / q$ be a convergent of the continued fraction expansion of the irrational number $\gamma$ such that $q>6 M$ and $\epsilon=\|\mu q\|-M\|\gamma q\|$, where $\mu$ is a real number and $\|\cdot\|$ denotes the distance from the nearest integer. If $\epsilon>0$, then there is no solution to the inequality

$$
0<m \gamma-n+\mu<A B^{-m}
$$

in positive integers $m$ and $n$ with

$$
\frac{\log (A q / \epsilon)}{\log B} \leq m<M
$$

\section{Proof of Theorem 2}

\subsection{The congruence method}

If $m=2$, then we get the solution

$$
L_{4} L_{5}=7 \cdot 11=7\left(\frac{10^{2}-1}{9}\right) .
$$

Assume that $k \geq 9$ and $m \geq 4$. Then, we have

$$
\nu_{2}\left(L_{n} \ldots L_{n+k-1}\right)=\nu_{2}\left(L_{n}\right)+\cdots+\nu_{2}\left(L_{n+k-1}\right) \geq 4 .
$$

Since $\nu_{2}\left(a\left(\frac{10^{m}-1}{9}\right)\right) \leq 3$, for $1 \leq a \leq 9$ an integer, then it yields an absurdity. So it follows that $k \leq 8$. 
If we focus on the 2-adic order of Lucas numbers and $a\left(\frac{10^{m}-1}{9}\right)$ in equation (2), then we have the several possible equations. We give the following tables containing the results obtained after taking the left and the right sides of equation (2) modulo $t$. As an example, it is obvious to see that $L_{6 n} L_{6 n+1} \equiv 2(\bmod 5)$. Namely, $\left\{L_{k}\right\}_{k \geq 0}$ has period 4 modulo 5 with the period being $2,1,3,4$. Since $6 n$ is even, it follows that either $6 n$ is a multiple of 4 , so then the two consecutive Lucas numbers are at the beginning of the period, namely the residues 2,1 with $2 \cdot 1 \equiv 2(\bmod 5)$, or $6 n \equiv 2(\bmod 4)$, in which case the Lucas numbers are the last two, namely the residues 3,4 with $3 \cdot 4 \equiv 2(\bmod 5)$.

It is obvious that $6 \frac{10^{m}-1}{9} \equiv 1(\bmod 5)$. Therefore, we arrive at a contradiction. Since the other cases can be proved by induction, we omit them. The sign $\dagger$ means a contradiction.

- Suppose that $n \equiv 0(\bmod 6)$.

\begin{tabular}{|c|c|c|c|c|c|}
\hline$k$ & $a$ & $t$ & $L_{n} \ldots L_{n+k-1}(\bmod t)$ & $a\left(\frac{10^{m}-1}{9}\right)(\bmod t)$ & Result \\
\hline \hline 2 & 2 & 16 & 2 or 10 & 14 & $\dagger$ \\
\hline 2 & 6 & 5 & 2 & 1 & $\dagger$ \\
\hline 4 & 8 & 10 & 4 & 8 & $\dagger$ \\
\hline 3 & 2 & 5 & 1 or 4 & 2 & $\dagger$ \\
\hline 3 & 6 & 8 & 6 & 2 & $\dagger$ \\
\hline 5 & 8 & 25 & 7 or 18 & 13 & $\dagger$ \\
\hline 6 & 8 & 25 & 18,3 or 23 & 13 & $\dagger$ \\
\hline
\end{tabular}

- Suppose that $n \equiv 1(\bmod 6)$. Then, we have:

\begin{tabular}{|c|c|c|c|c|c|}
\hline$k$ & $a$ & $t$ & $L_{n} \ldots L_{n+k-1}(\bmod t)$ & $a\left(\frac{10^{m}-1}{9}\right)(\bmod t)$ & Result \\
\hline \hline 2 & 1 & 8 & 3 & 7 & $\dagger$ \\
\hline 2 & 3 & 8 & 3 & 5 & $\dagger$ \\
\hline 2 & 5 & 10 & 3 & 5 & $\dagger$ \\
\hline 2 & 7 & 10 & 3 & 7 & $\dagger$ \\
\hline 2 & 9 & 10 & 3 & 9 & $\dagger$ \\
\hline 3 & 4 & 10 & 2 or 8 & 4 & $\dagger$ \\
\hline 4 & 4 & 39 & $24,33,33,24,6,27,6$ & $0,4,5,15,37,23$ & $\dagger$ \\
\hline & & & $19,34,28,12,11,24,8,17,3$, & & \\
5 & 4 & 37 & $17,8,24,11,12,28,34,19,36$, & & \\
& & & $1,18,3,9,25,26,13,29,20,34$, & & \\
\hline 6 & 8 & 30 & $20,29,13,26,25,9,3,18,1,36$ & & $18,8,28$ \\
\hline
\end{tabular}


- If $n \equiv 2(\bmod 6)$, then

\begin{tabular}{|c|c|c|c|c|c|}
\hline$k$ & $a$ & $t$ & $L_{n} \ldots L_{n+k-1} \quad(\bmod t)$ & $a\left(\frac{10^{m}-1}{9}\right)(\bmod t)$ & Result \\
\hline \hline 2 & 4 & 10 & 2 & 4 & $\dagger$ \\
\hline 3 & 4 & 39 & $\begin{array}{l}21,33,36,33,21,6 \\
33,18,6,3,6,18,33,6\end{array}$ & $15,37,23,0,4,5$ & $\dagger$ \\
\hline 4 & 4 & 39 & $6,24,33,33,24,6,27$ & $0,4,5,15,37,23$ & $\dagger$ \\
\hline 5 & \multirow{2}{*}{8} & 39 & $\begin{array}{l}21,27,36,21,36,27,21 \\
18,12,3,18,3,12,18\end{array}$ & $0,8,10,30,35,7$ & $\dagger$ \\
\hline 6 & 8 & 39 & $21,15,12,6,6,12,15$ & $0,8,10,30,35,7$ & $\dagger$ \\
\hline
\end{tabular}

- Now, assume that $n \equiv 3(\bmod 6)$. Then, we get following table.

\begin{tabular}{|c|c|c|c|c|c|}
\hline$k$ & $a$ & $t$ & $L_{n} \ldots L_{n+k-1}(\bmod t)$ & $a\left(\frac{10^{m}-1}{9}\right)(\bmod t)$ & Result \\
\hline \hline 2 & 4 & 5 & 3 & 4 & $\dagger$ \\
\hline 3 & 4 & 5 & 2 & 4 & $\dagger$ \\
\hline 4 & 8 & 10 & 4 & 8 & $\dagger$ \\
\hline 5 & 8 & 10 & 4 or 6 & 8 & $\dagger$ \\
\hline 6 & 8 & 10 & 2 & 8 & $\dagger$ \\
\hline
\end{tabular}

- Assume that $n \equiv 4(\bmod 6)$. Then, we obtain:

\begin{tabular}{|c|c|c|c|c|c|}
\hline$k$ & $a$ & $t$ & $L_{n} \ldots L_{n+k-1}(\bmod t)$ & $a\left(\frac{10^{m}-1}{9}\right)(\bmod t)$ & Result \\
\hline \hline 2 & 1 & 5 & 2 & 1 & $\dagger$ \\
\hline 2 & 3 & 20 & 17 & 13 & $\dagger$ \\
\hline 2 & 5 & 5 & 2 & 0 & $\dagger$ \\
\hline 2 & 7 & 8 & 5 & 1 & $\dagger$ \\
\hline 2 & 9 & 9 & 5 or 6 & 0 & $\dagger$ \\
\hline 3 & 2 & 8 & 2 & 6 & $\dagger$ \\
\hline 3 & 6 & - & - & 2 & possible \\
\hline 4 & 2 & 20 & 14 & 6 & $\dagger$ \\
\hline 4 & 6 & 20 & 14 & 22 & $\dagger$ \\
\hline 5 & 2 & 25 & 18,7 & 16 & $\dagger$ \\
\hline 5 & 6 & 25 & 18,7 & 13 & $\dagger$ \\
\hline 6 & 8 & 25 & $18,23,3$ & & \\
\hline
\end{tabular}


- Finally, if $n \equiv 5(\bmod 6)$, then

\begin{tabular}{|c|c|c|c|c|c|}
\hline$k$ & $a$ & $t$ & $L_{n} \ldots L_{n+k-1}(\bmod t)$ & $a\left(\frac{10^{m}-1}{9}\right)(\bmod t)$ & Result \\
\hline \hline 2 & 2 & 20 & 18 & 2 & $\dagger$ \\
\hline 2 & 6 & 24 & 6,22 & 18 & $\dagger$ \\
\hline 3 & 2 & 25 & $\begin{array}{l}13,12,8,2,8, \\
12,13,17,23,17\end{array}$ & $\dagger$ \\
\hline 3 & 6 & 16 & 14 & 22 & $\dagger$ \\
\hline 4 & 2 & 15 & 9 & 12,2 or 7 & $\dagger$ \\
\hline 4 & 6 & 15 & 9 & 6 & $\dagger$ \\
\hline 5 & 8 & 25 & 1,24 & 13 & $\dagger$ \\
\hline 6 & 8 & 25 & $7,2,22$ & 13 & $\dagger$ \\
\hline
\end{tabular}

From all the tables, we see that the equation $L_{n} L_{n+1} L_{n+2}=\frac{6}{9}\left(10^{m}-1\right)$ is possible if $n \equiv 4$ (mod 6). We solve this equation in the next subsection by Baker's method.

\subsection{The equation $L_{n} L_{n+1} L_{n+2}=\frac{6}{9}\left(10^{m}-1\right)$}

In this subsection, we prove that the equation

$$
L_{n} L_{n+1} L_{n+2}=\frac{6}{9}\left(10^{m}-1\right)=\frac{2}{3}\left(10^{m}-1\right)
$$

has no solution with positive integers $n$ and $m \geq 3$. Combining the Binet formula for Lucas numbers with the fact $L_{n} L_{n+1} L_{n+2}=L_{3 n+3}+(-1)^{n} 2 L_{n+1}$, we get

$$
\alpha^{3 n+3}-\frac{2}{3} 10^{m}=\frac{-2}{3}-\beta^{3 n+3}-(-1)^{n}\left(2 \alpha^{n+1}+2 \beta^{n+1}\right) .
$$

Thus, we obtain

$$
\begin{aligned}
\left|1-\frac{2}{3} 10^{m} \alpha^{-(3 n+3)}\right| & \leq \frac{2}{3 \alpha^{3 n+3}}+\frac{|\beta|^{3 n+3}}{\alpha^{3 n+3}}+\frac{2}{\alpha^{2 n+2}}+\frac{2|\beta|^{n+1}}{\alpha^{3 n+3}} \\
& <\frac{4}{\alpha^{3 n+3}}+\frac{2}{\alpha^{2 n+2}}<\frac{6}{\alpha^{2 n+2}} .
\end{aligned}
$$

Let

$$
\Lambda:=\frac{2}{3} \alpha^{-(3 n+3)} 10^{m}-1 .
$$

In order to apply Lemma 2, we take

$$
\gamma_{1}:=\frac{2}{3}, \gamma_{2}:=\alpha, \gamma_{3}:=10, b_{1}:=1, b_{2}:=3 n+3, b_{3}:=m
$$

For this choice, we have $D=2, t=3, B=3 n+3, A_{1}:=0.16, A_{2}:=0.5$, and $A_{3}:=2.31$. As $\alpha, 10$, and $2 / 3$ are multiplicatively independent, we have $\Lambda \neq 0$. We combine Lemma 2 and the inequality (5) to obtain

$$
\exp (K(1+\log (3 n+3)))<|\Lambda|<\frac{6}{\alpha^{2 n+2}},
$$


where $K:=-1.4 \cdot 30^{6} \cdot 3^{4.5} \cdot 4(1+\log 2) \cdot 0.16 \cdot 0.5 \cdot 2.31$. Inequality (6) yields that

$$
n<5.87 \cdot 10^{12}
$$

By the estimates for Lucas numbers given by (3) and equation (4), we have $10^{m}<\alpha^{3 n+6}$ and then

$$
\left|1-\frac{2}{3} 10^{m} \alpha^{-(3 n+3)}\right|<\frac{6}{\alpha^{2 n+2}}<\frac{73}{\left(10^{2 / 3}\right)^{m}} .
$$

Let $z:=m \log 10-(3 n+3) \log \alpha+\log \frac{2}{3}$. Thus,

$$
\left|1-e^{z}\right|<\frac{73}{\left(10^{2 / 3}\right)^{m}}
$$

holds. It is obvious that $z \neq 0$. If $z>0$, then

$$
0<z \leq\left|1-e^{z}\right|<\frac{73}{\left(10^{2 / 3}\right)^{m}}
$$

Otherwise $(z<0)$, we get

$$
0<|z| \leq e^{|z|}-1=e^{|z|}\left|1-e^{z}\right|<\frac{146}{\left(10^{2 / 3}\right)^{m}}
$$

where we use the fact $\left|1-e^{z}\right|<\frac{1}{2}$. In any case, we obtain that

$$
\begin{aligned}
0 & <\left|m \log 10-(3 n+3) \log \alpha+\log \frac{2}{3}\right| \\
& <\frac{146}{\left(10^{2 / 3}\right)^{m}}<\frac{146}{(4.6)^{m}} .
\end{aligned}
$$

Dividing by $3 \log \alpha$, we get

$$
|m \gamma-n+\mu|<102 \cdot(4.6)^{-m}
$$

with $\gamma:=\frac{\log 10}{3 \log \alpha}$ and $\mu:=\frac{\log \left(\frac{2}{3}\right)}{3 \log \alpha}$. Let $q_{t}$ be the denominator of the $t$-th convergent of the continued fraction of $\gamma$. Taking $M:=5.87 \cdot 10^{12}$, we have

$$
q_{32}=109143857145934>6 M,
$$

and then $\epsilon:=\left\|\mu q_{32}\right\|-M\left\|\gamma q_{32}\right\|>0$. The conditions of Lemma 3 are fulfilled for $A:=102$ and $B:=4.6$. Then, there are no solutions for the interval

$$
\left[\left\lfloor\frac{\log \left(\frac{102 \cdot q_{32}}{\epsilon}\right)}{\log B}\right\rfloor+1, M\right)=\left[26,5.87 \cdot 10^{12}\right) .
$$

Therefore, it remains to check equation (2) for $3 \leq m \leq 25$. For this, we use a program written in Mathematica and see that there are no solutions of the equation

$$
L_{n} L_{n+1} L_{n+2}=\frac{6}{9}\left(10^{m}-1\right) .
$$

This completes the proof of Theorem 2 . 


\section{References}

[1] Bugeaud, Y., Mignotte, M., \& Siksek, S. (2006) Classical and modular approaches to exponential Diophantine equations, I. Fibonacci and Lucas powers, Ann. of Math., 163, 969-1018.

[2] Dujella, A., A. Pethö (1998) A generalization of a theorem of Baker and Davenport, Quart. J. Math. Oxford Ser. (2), 49 (195), 291-306.

[3] Koshy, T. (2003) Fibonacci and Lucas Numbers with Applications, Wiley.

[4] Lengyel, T. (1995) The order of the Fibonacci and Lucas numbers, Fibonacci Quart., 33(3), 234-239.

[5] Luca, F. (2000) Fibonacci and Lucas numbers with only one distinct digit, Portugal. Math. $50,243-254$.

[6] Luca, F., T. N. Shorey (2005) Diophantine equations with product of consecutive terms in Lucas sequences, J. Number Theory, 114, 298-311.

[7] Marques, D., \& Togbé, A. (2012) On repdigits as product of consecutive Fibonacci numbers. Rend. Istit. Mat. Univ. Trieste, 44, 393-397.

[8] Matveev, E. M. (2000) An explicit lower bound for a homogeneous linear form in logarithms of algebraic numbers. II, Izv. Ross. Akad. Nauk Ser. Mat. 64(6), 125-180; translation in Izv. Math. 64 (200), 6, 1217-1269. 